\title{
Dinámica de precios y valoración de activos contingentes en mercados con riesgo de liquidez
}

\author{
Price dynamics and valuation of contingent \\ assets in markets with liquidity risk
}

John Freddy Moreno Trujillo*

* Estudiante de Doctorado en Ciencias Económicas. Magíster en Matemática Aplicada. Docente-Investigador, CIPE-ODEON, Universidad Externado de Colombia, Bogotá (Colombia). [jhon.moreno@uexternado.edu.co], [ORCID: 0000-0002-2772-6931].

Artículo recibido el 15 de noviembre de 2020.

Aceptado el 15 de diciembre de 2020.

Para citar este artículo:

Moreno Trujillo, J. F. (2020). Modelo para la dinámica del precio de activos riesgosos en mercados con liquidez. ODEON, 19, 2020, 153-180.

DOI: https://doi.org/10.18601/17941113.n19.06 


\section{Resumen}

Se consideran modelos de mercado en donde se incorpora un factor de liquidez asociado a la dinámica de los precios y a las estrategias de negociación de los agentes. Se estudia el caso en el cual el factor de liquidez es una función determinística del precio y otro en donde este factor es estocástico descrito por un proceso Cox-Ingersoll-Ross. Se consideran diferentes tipos de estrategias de negociación y se establecen de forma explícita las ecuaciones diferenciales estocásticas para la dinámica de los precios, así como las ecuaciones diferenciales parciales no lineales de valoración de activos contingentes correspondientes.

Palabras clave: factor de liquidez; liquidez estocástica; dinámica de precios; ecuación de valoración no lineal.

Clasificación JEL: C02, C60, C63. 


\begin{abstract}
Market models are considered in which a liquidity factor associated with price dynamics and the agents' trading strategies is incorporated. The case is studied in which the liquidity factor is a deterministic function of the price and another in which this factor is stochastic described by a Cox-Ingersoll-Ross process. Different types of trading strategies are considered and the stochastic differential equations for price dynamics as well as the corresponding nonlinear partial differential equations for the valuation of contingent assets are explicitly established
\end{abstract}

Key words: Liquidity factor; stochastic liquidity; price dynamics; non-linear valuation equation.

JEL classification: C02, C60, C63. 


\section{Introducción}

Los modelos clásicos de valoración de activos contingentes están fundamentados en una serie de supuestos simplificadores sobre la dinámica y el funcionamiento de los mercados, en particular, en el ampliamente conocido modelo de BlackScholes (Black \& Scholes, 1973) se considera que los mercados de todos los activos son libres de fricciones, lo que implica que son perfectamente líquidos y competitivos, lo cual permite que los agentes del mercado puedan establecer estrategias de cobertura y replicación sin afectar los precios de los activos subyacentes. Aunque este tipo de supuestos permite encontrar expresiones analíticas relativamente sencillas de valoración, no capturan la realidad de los mercados, pues es ampliamente conocido que los agentes establecen sus estrategias de negociación considerando un inherente riesgo de liquidez, producto de la disponibilidad limitada en volumen y tiempo de los activos (Tripathi et al., 2019).

En años recientes se ha observado un importante esfuerzo por desarrollar modelos que incorporen los efectos de la iliquidez en la dinámica de los precios y en la valoración de activos contingentes. Las dos principales corrientes de investigación a este respecto son: 1) la discusión y caracterización de los hallazgos empíricos sobre los efectos de la iliquidez; 2) el desarrollo de modelos teóricos que consideran la iliquidez en el proceso de valoración de activos. En los dos casos se distingue entre modelos de liquidez agregada y modelos de liquidez específica. En el primer caso, se considera que la liquidez de todo el mercado fluctúa con el tiempo y que esta tiene un impacto diferente en conjuntos de activos distintos. Algunos trabajos que consideran este enfoque son Amihud (2002), Amihud y Mendelson (1986), Chordia et al. (2000) y Chordia et al. (2001). En el segundo caso se estudia la liquidez específica de los activos, ya sea modelando explícitamente su impacto en el precio 
por negociaciones de diferente volumen, como en los trabajos de Cetin et al. (2010), Ronnie et al. (1998) y Wilmott y Schönbucher (2000), o introduciendo costos de transacción en modelos de valoración de activos, donde los trabajos de Lo et al. (2004), Vayanos (2004) y Acharya \& Pedersen (2005) son algunos ejemplos.

Existe una larga lista de hechos estilizados asociados a la iliquidez del mercado. Por ejemplo, Amihud \& Mendelson (1986) y Amihud (2002) analizan los efectos de la iliquidez agregada y específica de las acciones sobre el exceso de rentabilidad; ambos encuentran que el exceso de rentabilidad aumenta con la falta de liquidez. Pástor \& Stambaugh (2003) proporcionan evidencia de que la iliquidez agregada tiende a ser baja cuando la volatilidad del mercado es alta, al medir el índice de Sharpe de una cartera de muestra antes y después de choques de liquidez.

En el presente documento se estudia un mercado en el cual la dinámica de los precios incorpora un factor de liquidez asociado a las estrategias de negociación de los agentes. Se consideran dos casos, en el primero el factor de liquidez se asume como una función determinística del precio del activo riesgoso; en el segundo el factor de liquidez es estocástico, siguiendo un proceso del tipo Cox-Ingersoll-Ross (CIR). En los dos casos se consideran algunas formas específicas para la estrategia de negociación o cobertura, en particular se considera que los agentes siguen estrategias de cobertura delta.

En cada caso se estudia la relación entre la dinámica de los precios y las estrategias de negociación, y además se establecen las ecuaciones diferenciales parciales (EDP) de valoración de activos contingentes correspondientes, donde se encuentra que estas EDP resultan ser extensiones no lineales de la EDP de Black-Scholes.

En la sección 1 se realiza una breve presentacion del clásico modelo de mercado Black-Scholes y de la correspondiente EDP de valoración. En la sección 2 
se presenta un modelo de mercado en el cual la dinámica del precio de los activos riesgosos incorpora un factor de liquidez determinístico relacionado con el precio del activo y con las estrategias de negociación de los agentes, y se deduce la correspondiente EDP de valoración de activos contingentes. En esta sección también se considera el caso en el cual la estrategia de negociación tiene un componente de aleatoriedad, en particular se asume que esta estrategia es descrita por un movimiento Browniano aritmético. En la sección 3 se considera el caso en el cual el factor de liquidez del mercado es estocástico, se caracteriza la dinámica de los precios en este contexto y se establece la EDP de valoración. Finalmente, se presentan algunas conclusiones y futuras extensiones.

\section{Modelo de mercado con liquidez perfecta}

Se considera un espacio de probabilidad de referencia $\left(\Omega, \mathcal{F}, \mathbb{P},\left(\mathcal{F}_{t}\right)_{t \in[0, T]}\right)$ para algún horizonte de tiempo fijo $T$, y se asumen las condiciones necesarias sobre los coeficientes de las ecuaciones diferenciales estocásticas utilizadas para garantizar la existencia de sus soluciones. Se considera un mercado financiero libre de arbitraje y compuesto por:

i) Un activo libre de riesgo, que representa el valor del efectivo en el mercado, y que permite a los agentes tomar prestado o invertir a una tasa de interés $r$ constante y conocida. Si una cantidad $B_{0}$ se invierte (o se toma prestada) en este activo en el instante $t=0$, el monto que se recibe (o paga) en algún instante $t \geq 0$ estará dado por:

$$
B_{t}=B_{0} e^{r t} \quad ; \quad d B_{t}=r B_{t} d t
$$

ii) Un activo riesgoso con precio en $t$ denotado por $S_{t}$, con tasa de rentabilidad 
$\alpha$ y volatilidad $\sigma>0$ constantes, que sigue un movimiento Browniano geométrico de la forma:

$$
S_{t}=S_{0} e^{\left(\alpha-\frac{\sigma^{2}}{2}\right) t+\sigma W_{t}} \quad ; \quad d S_{t}=\alpha S_{t} d t+\sigma S_{t} d W_{t}
$$

Se asume que el mercado de los dos activos es perfectamente líquido, es decir, los agentes pueden negociar (tomar posiciones largas o cortas) en cualquier cantidad de estos activos de forma inmediata y sin alterar su precio.

iii) Un activo contingente (derivado) pactado sobre el activo riesgoso, con valor en $t$ denotado por $V \equiv V\left(t, S_{t}\right)$, con vencimiento en $T>0$ y $V\left(T, S_{T}\right)=\Phi\left(S_{T}\right)$. Aplicando el lema de Itô se tiene que el proceso de precio del activo contingente satisface:

$$
d V=\left(\frac{\partial V}{\partial t}+\alpha S_{t} \frac{\partial V}{\partial S_{t}}+\frac{1}{2} \sigma^{2} S_{t}^{2} \frac{\partial^{2} V}{\partial S_{t}^{2}}\right) d t+\sigma S_{t} \frac{\partial V}{\partial S_{t}} d W_{t}
$$

Se busca determinar $V \equiv V\left(t, S_{t}\right)$, para lo cual se considera un portafolio de cobertura $\Pi_{t}$ autofinanciado, compuesto por: $f_{t}$ unidades del activo riesgoso y $\gamma_{t}$ unidades del activo contingente, de forma que:

$$
\Pi_{t}=f_{t} S_{t}+\gamma_{t} V \quad \text { y } \quad d \Pi_{t}=f_{t} d S_{t}+\gamma_{t} d V
$$

De las expresiones (2) y (3) se sigue que:

$$
\begin{aligned}
d \Pi_{t} & =f_{t} d S_{t}+\gamma_{t} d V \\
& =f_{t}\left(\alpha S_{t} d t+\sigma S_{t} d W_{t}\right)+\gamma_{t}\left[\left(\frac{\partial V}{\partial t}+\alpha S_{t} \frac{\partial V}{\partial S_{t}}+\frac{1}{2} \sigma^{2} S_{t}^{2} \frac{\partial^{2} V}{\partial S_{t}^{2}}\right) d t+\sigma S_{t} \frac{\partial V}{\partial S_{t}} d W_{t}\right] \\
& =\alpha S_{t}\left(f_{t}+\gamma_{t} \frac{\partial V}{\partial S_{t}}\right) d t+\sigma S_{t}\left(f_{t}+\gamma_{t} \frac{\partial V}{\partial S_{t}}\right) d W_{t}+\gamma_{t}\left(\frac{\partial V}{\partial t}+\frac{1}{2} \sigma^{2} S_{t}^{2} \frac{\partial^{2} V}{\partial S_{t}^{2}}\right) d t
\end{aligned}
$$


Si la estrategia de negociación del agente se establece de forma que el coeficiente del término de difusión en (5) se anule, es decir, $f_{t}+\gamma_{t} \frac{\partial V}{\partial S_{t}}=0$, se tiene un portafolio sin riesgo. Debido a la ausencia de oportunidades de arbitraje en el mercado, esta estrategia debe generar la misma rentabilidad que si se invierte el valor inicial de portafolio a la tasa libre de riesgo $r$.

Existen infinitas estrategias que permiten generar un portafolio de cobertura sin riesgo, pero dado que el objetivo es determinar cuál debe ser la estrategia del agente para cubrir una unidad del activo contingente, nos concentramos en establecer el número de unidades del activo riesgoso en las que debe tomar posición $\left(f_{t}\right)$ considerando que $\gamma_{t}=1$, es decir, buscamos determinar cuál debe ser la posición en el activo riesgoso que permite cubrir una posición larga en una unidad del activo contingente.

Si $\gamma_{t}=1$, entonces $f_{t}=-\frac{\partial V}{\partial S_{t}}$, es decir, la cobertura de la posición larga en una unidad del activo contingente se consigue tomando una posición corta en $\frac{\partial V}{\partial S_{t}}$ unidades del activo riesgoso subyacente, o, dicho de otra forma, para cubrir una posición corta en $\frac{\partial V}{\partial S_{t}}$ unidades del activo riesgoso es necesario tomar posición larga en una unidad del activo contingente. Siguiendo esta estrategia, la ecuación (5) se transforma en:

$$
d \Pi_{t}=\left(\frac{\partial V}{\partial t}+\frac{1}{2} \sigma^{2} S_{t}^{2} \frac{\partial^{2} V}{\partial S_{t}^{2}}\right) d t
$$

expresión que, como se mencionó, por no arbitraje debe ser igual al resultado de invertir en el valor inicial del portafolio a la tasa libre de riesgo, es decir, invertir:

$$
\Pi_{t}=f_{t} S_{t}+\gamma_{t} V=-\frac{\partial V}{\partial S_{t}} S_{t}+V
$$

a la tasa $r$, con lo cual: 


$$
d \Pi_{t}^{(r)}=\left(-\frac{\partial V}{\partial S_{t}} S_{t}+V\right) r d t
$$

Igualando las expresiones (6) y (8) se tiene la ecuación diferencial parcial (EDP) de valoración de Black-Scholes:

$$
\frac{\partial V}{\partial t}+r S_{t} \frac{\partial V}{\partial S_{t}}+\frac{1}{2} \sigma^{2} S_{t}^{2} \frac{\partial^{2} V}{\partial S_{t}^{2}}-r V=0
$$

junto con la condición de frontera $V\left(T, S_{T}\right)=\Phi\left(S_{T}\right)$. Es bien conocido que esta EDP puede ser resuelta de forma analítica para un conjunto importante de activos contingentes, y que, en general, su solución puede aproximarse mediante métodos numéricos como diferencias finitas.

\section{Modelo de mercado con liquidez determinística}

Se considera ahora una extensión del modelo de mercado descrito en la sección anterior, en la cual la dinámica del precio del activo riesgoso incorpora un factor de liquidez. La ecuación diferencial estocástica (EDE) que satisface el proceso de precio de activos riesgosos en este caso es:

$$
d S_{t}=\alpha S_{t} d t+\sigma S_{t} d W_{t}+\lambda\left(t, S_{t}\right) d f\left(t, S_{t}\right)
$$

donde: $\lambda\left(t, S_{t}\right)$ denota el factor de liquidez del mercado, y $f\left(t, S_{t}\right)$ describe el número de unidades del activo riesgoso que posee el agente como parte de su estrategia de negociación. De esta forma, el término $\lambda\left(t, S_{t}\right) d f\left(t, S_{t}\right)$ describe el impacto sobre el precio del activo riesgoso de un cambio en el número de unidades de dicho activo ponderado por el factor de liquidez del mercado. El factor de liquidez $\lambda\left(t, S_{t}\right)$ expresa una presión a la baja en el precio del activo cuando el agente 
vende unidades $\left(d f\left(t, S_{t}\right)<0\right)$ y una presión al alza cuando compra $\left(d f\left(t, S_{t}\right)>0\right)$. En el caso particular de este modelo con liquidez determinística asumiremos que $\lambda\left(t, S_{t}\right)=\lambda S_{t}$, donde $\lambda>0$ es constante. Se tiene entonces que:

$$
d S_{t}=\alpha S_{t} d t+\sigma S_{t} d W_{t}+\lambda S_{t} d f\left(t, S_{t}\right)
$$

Se puede ver que, manteniendo sin cambios los demás términos de la expresión (11), si $d f\left(t, S_{t}\right)>0$ (lo que representa la compra de unidades por parte del agente) el precio tiende a subir, mientras que si $d f\left(t, S_{t}\right)<0$ (lo que representa la venta de unidades por parte del agente) el precio tiende a bajar. Desde luego, una estrategia de comprar y mantener la posición hasta el final, $d f\left(t, S_{t}\right)=0$, no afecta los precios.

Asumiendo que las estrategias de negociación seguidas por los agentes satisfacen que $f\left(t, S_{t}\right) \in C^{1,2}\left([0, T] \times \mathbb{R}_{+}\right)$, aplicando la fórmula de Itô se tiene que:

$$
d f\left(t, S_{t}\right)=\frac{\partial f}{\partial t} d t+\frac{\partial f}{\partial S_{t}} d S_{t}+\frac{1}{2} \frac{\partial^{2} f}{\partial S_{t}^{2}}\left(d S_{t}\right)^{2}
$$

y reemplazando en la expresión (11):

$$
\begin{aligned}
d S_{t} & =\alpha S_{t} d t+\sigma S_{t} d W_{t}+\lambda S_{t} d f\left(t, S_{t}\right) \\
d S_{t} & =\alpha S_{t} d t+\sigma S_{t} d W_{t}+\lambda S_{t}\left(\frac{\partial f}{\partial t} d t+\frac{\partial f}{\partial S_{t}} d S_{t}+\frac{1}{2} \frac{\partial^{2} f}{\partial S_{t}^{2}}\left(d S_{t}\right)^{2}\right) \\
d S_{t} & =\alpha S_{t} d t+\sigma S_{t} d W_{t}+\lambda S_{t} \frac{\partial f}{\partial t} d t+\lambda S_{t} \frac{\partial f}{\partial S_{t}} d S_{t}+\frac{1}{2} \lambda S_{t} \frac{\partial^{2} f}{\partial S_{t}^{2}}\left(d S_{t}\right)^{2} \\
d S_{t}-\lambda S_{t} \frac{\partial f}{\partial S_{t}} d S_{t} & =\left(\alpha S_{t}+\lambda S_{t} \frac{\partial f}{\partial t}\right) d t+\frac{\lambda S_{t}}{2} \frac{\partial^{2} f}{\partial S_{t}^{2}}\left(d S_{t}\right)^{2}+\sigma S_{t} d W_{t} \\
d S_{t}\left(1-\lambda S_{t} \frac{\partial f}{\partial S_{t}}\right) & =\left(\alpha S_{t}+\lambda S_{t} \frac{\partial f}{\partial t}\right) d t+\frac{\lambda S_{t}}{2} \frac{\partial^{2} f}{\partial S_{t}^{2}}\left(d S_{t}\right)^{2}+\sigma S_{t} d W_{t}
\end{aligned}
$$

elevando al cuadrado esta expresión y considerando que $d t \rightarrow 0$ se tiene que: 


$$
\left(d S_{t}\right)^{2}\left(1-\lambda S_{t} \frac{\partial f}{\partial S_{t}}\right)^{2}=\underbrace{\left(\frac{\lambda S_{t}}{2} \frac{\partial^{2} f}{\partial S_{t}^{2}}\left(d S_{t}\right)^{2}\right)^{2}}_{o(t) \rightarrow 0}+\sigma^{2} S_{t}^{2} d t
$$

con lo cual,

$$
\left(d S_{t}\right)^{2}=\frac{\sigma^{2} S_{t}^{2} d t}{\left(1-\lambda S_{t} \frac{\partial f}{\partial S_{t}}\right)^{2}}
$$

y de la expresión (13) se sigue que:

$$
\begin{aligned}
d S_{t}\left(1-\lambda S_{t} \frac{\partial f}{\partial S_{t}}\right) & =\left(\alpha S_{t}+\lambda S_{t} \frac{\partial f}{\partial t}\right) d t+\frac{\lambda S_{t}}{2} \frac{\partial^{2} f}{\partial S_{t}^{2}}\left(d S_{t}\right)^{2}+\sigma S_{t} d W_{t} \\
d S_{t}\left(1-\lambda S_{t} \frac{\partial f}{\partial S_{t}}\right) & =\left(\alpha S_{t}+\lambda S_{t} \frac{\partial f}{\partial t}\right) d t+\frac{\lambda S_{t}}{2} \frac{\partial^{2} f}{\partial S_{t}^{2}}\left(\frac{\sigma^{2} S_{t}^{2} d t}{\left(1-\lambda S_{t} \frac{\partial f}{\partial S_{t}}\right)^{2}}\right)+\sigma S_{t} d W_{t} \\
d S_{t}\left(1-\lambda S_{t} \frac{\partial f}{\partial S_{t}}\right) & =\left[\alpha S_{t}+\lambda S_{t}\left(\frac{\partial f}{\partial t}+\frac{1}{2}\left(\frac{\sigma S_{t}}{1-\lambda S_{t} \frac{\partial f}{\partial S_{t}}}\right)^{2} \frac{\partial^{2} f}{\partial S_{t}^{2}}\right)\right] d t+\frac{\sigma S_{t}}{1-\lambda S_{t} \frac{\partial f}{\partial S_{t}}} d W_{t} \\
d S_{t} & =\frac{1}{\left(1-\lambda S_{t} \frac{\partial f}{\partial S_{t}}\right)}\left[\alpha S_{t}+\lambda S_{t}\left(\frac{\partial f}{\partial t}+\frac{1}{2}\left(\frac{\sigma S_{t}}{1-\lambda S_{t} \frac{\partial f}{\partial S_{t}}}\right)^{2} \frac{\partial^{2} f}{\partial S_{t}^{2}}\right)\right] d t+\frac{\sigma S_{t}}{1-\lambda S_{t} \frac{\partial f}{\partial S_{t}}} d W_{t}
\end{aligned}
$$

y denotando por:

$$
\begin{gathered}
\hat{\alpha}=\frac{1}{\left(1-\lambda S_{t} \frac{\partial f}{\partial S_{t}}\right)}\left[\alpha S_{t}+\lambda S_{t}\left(\frac{\partial f}{\partial t}+\frac{1}{2}\left(\frac{\sigma S_{t}}{1-\lambda S_{t} \frac{\partial f}{\partial S_{t}}}\right)^{2} \frac{\partial^{2} f}{\partial S_{t}^{2}}\right)\right] \\
\hat{\sigma}=\frac{\sigma S_{t}}{1-\lambda S_{t} \frac{\partial f}{\partial S_{t}}}
\end{gathered}
$$

se tiene que la dinámica de los precios en este modelo es:

$$
d S_{t}=\hat{\alpha} d t+\hat{\sigma} d W_{t}
$$

donde $1-\lambda S_{t} \frac{\partial f}{\partial S_{t}}>0$ para garantizar la consistencia de los precios. 


\subsection{Estrategias de negociación}

En los mercados financieros actuales es posible encontrar muy diversas estrategias de negociación. A continuación se consideran algunos ejemplos para el caso en el cual la estrategia puede ser expresada como una función de $t$ y $S_{t}\left(f\left(t, S_{t}\right)\right)$.

- Comprar y mantener: esta estrategia consiste en comprar una unidad del activo y no negociarla más en ningún momento, $f\left(t, S_{t}\right)=1$.

- Órdenes límites: en el caso de una orden de venta con precio de ejercicio $\bar{S}$ la estrategia es vender una unidad del activo si el precio alcanza o supera el precio $\bar{S}$ y luego no hacer nada, es decir, antes de vender la estrategia es:

$$
f\left(t, S_{t}\right)=H\left(\bar{S}-S_{t}\right)= \begin{cases}0 & \text { si } \bar{S}-S_{t} \leq 0 \\ 1 & \text { si } \bar{S}-S_{t}>0\end{cases}
$$

y después de la venta es $f\left(t, S_{t}\right)=0$. En el caso de una orden de compra con precio de ejercicio $\bar{S}$ la estrategia es comprar una unidad del activo si el precio alcanza o supera el precio $\bar{S}$ y luego no hacer nada, es decir, antes de la compra la estrategia es:

$$
f\left(t, S_{t}\right)=H\left(S_{t}-\bar{S}\right)= \begin{cases}0 & \text { si } S_{t}-\bar{S} \leq 0 \\ 1 & \text { si } S_{t}-\bar{S}>0\end{cases}
$$

y después de la compra es $f\left(t, S_{t}\right)=1$.

- Negociando a través de la barrera: en este caso $f\left(t, S_{t}\right)=H\left(S_{t}-\bar{S}\right)$, es decir, cuando $S_{t}>\bar{S}$ tener una unidad del activo, y cuando $S_{t}<\bar{S}$ no tener unidades del activo. En este caso, cuando el precio del activo supera el precio $\bar{S}$ desde valores inferiores se compra una unidad y si los cruza desde valores superiores se vende. 
Para este tipo de estrategias se tiene que $\frac{\partial f}{\partial S_{t}}=0 \mathrm{y} \frac{\partial f}{\partial t}=0$, con lo que $\hat{\alpha}=\alpha$ y $\hat{\sigma}=\sigma$, es decir, el proceso de precios es el clásico movimiento Browniano geométrico del modelo Black-Scholes.

- Estrategias basadas en optimización: este tipo de estrategias son las que surgen como solución a problemas de control óptimo estocástico relacionados con la selección de estrategias de inversión y consumo por parte de un gente con preferencias modeladas vía su función de utilidad. Un ejemplo clásico de este tipo de estrategias se encuentra en Merton \& Samuelson (1990), donde se analizan reglas de inversión óptimas para agentes con funciones de utilidad con aversión absoluta al riesgo constante en el contexto de un mercado con precios lognormales.

En general, las estrategias de inversión óptima encontradas en este caso son de la forma:

$$
f\left(t, S_{t}\right)=\theta_{0} e^{-\theta_{1} t} S_{t}^{-\theta_{2}}
$$

donde $\theta_{0}, \theta_{1}, \theta_{2}$ son constantes positivas que dependen de la especificación del modelo. Estrategias más complejas pueden ser derivadas al considerar otras especificaciones de la función de utilidad de los agentes o del conjunto de oportunidades de inversión, planteando el problema de optimización y resolviendo la correspondiente ecuación de Hamilton-Jacobi-Bellman.

La figura 1 muestra la representación de esta estrategia en diferentes instantes de tiempo y para distintos valores del $S_{t}$. En la figura de la izquierda $\theta_{0}=10 ; \theta_{1}=$ $\theta_{2}=1$, lo que representa una estrategia en la cual el agente adquiere (largo) más unidades del activo si el precio está bajo. En la figura de la derecha $\theta_{0}=-1 ; \theta_{1}=$ $\theta_{2}=1$, en este caso la estrategia indica que el agente vende (corto) más unidades del activo si el precio está bajo. 
Figura 1: Estrategias de negociación
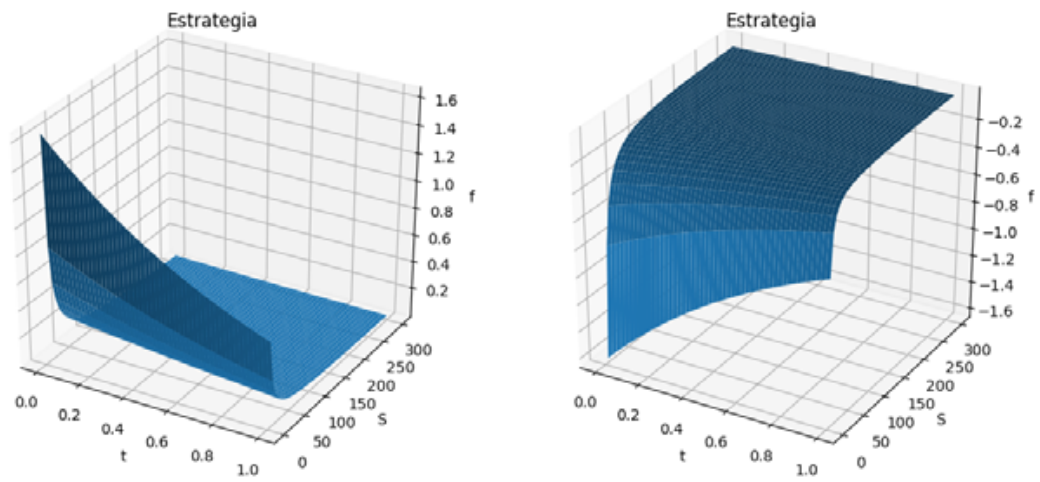

Para estrategias de inversión como las descritas en (21) se tiene que:

$\frac{\partial f}{\partial t}=-\left(\theta_{0} \theta_{1}\right) e^{-\theta_{1} t} S_{t}^{-\theta_{2}} \quad ; \quad \frac{\partial f}{\partial S_{t}}=-\left(\theta_{0} \theta_{2}\right) e^{-\theta_{1} t} S_{t}^{-\left(\theta_{2}+1\right)} \quad ; \quad \frac{\partial^{2} f}{\partial S_{t}^{2}}=\left(\theta_{0} \theta_{2}\right)\left(\theta_{2}+1\right) e^{-\theta_{1} t} S_{t}^{-\left(\theta_{2}+2\right)}$

de donde se sigue que la expresión para la dinámica de los precios es:

$$
d S_{t}=\hat{\alpha} d t+\hat{\sigma} d W_{t}
$$

con:

$$
\begin{gathered}
\hat{\sigma}=\frac{\sigma S_{t}}{1+\lambda \theta_{0} \theta_{2} e^{-\theta_{1} t} S_{t}^{-\theta_{2}}} \\
\hat{\alpha}=\left(\frac{1}{1+\lambda \theta_{0} \theta_{2} e^{-\theta_{1} t} S_{t}^{-\theta_{2}}}\right)\left\{\alpha S_{t}+\lambda \theta_{0} e^{-\theta_{1} t} S_{t}^{1-\theta_{2}}\left[\frac{\theta_{2}\left(\theta_{2}+1\right)}{2}\left(\frac{\sigma}{1+\lambda \theta_{0} \theta_{2} e^{-\theta_{1} t} S_{t}^{-\theta_{2}}}\right)^{2}\right]\right\}
\end{gathered}
$$

La figura 2 muestra 50 trayectorias simuladas del precio del activo considerando que el agente sigue las estrategias representadas en la figura 1 respectivamente. Se puede ver que 
si la estrategia del agente es comprar (largo) empuja el precio a la alza, mientras que si la estrategia del agente es vender (corto) empuja el precio a la baja.

Figura 2: Trayectorias simuladas de los precios bajo diferentes estrategias de negociación
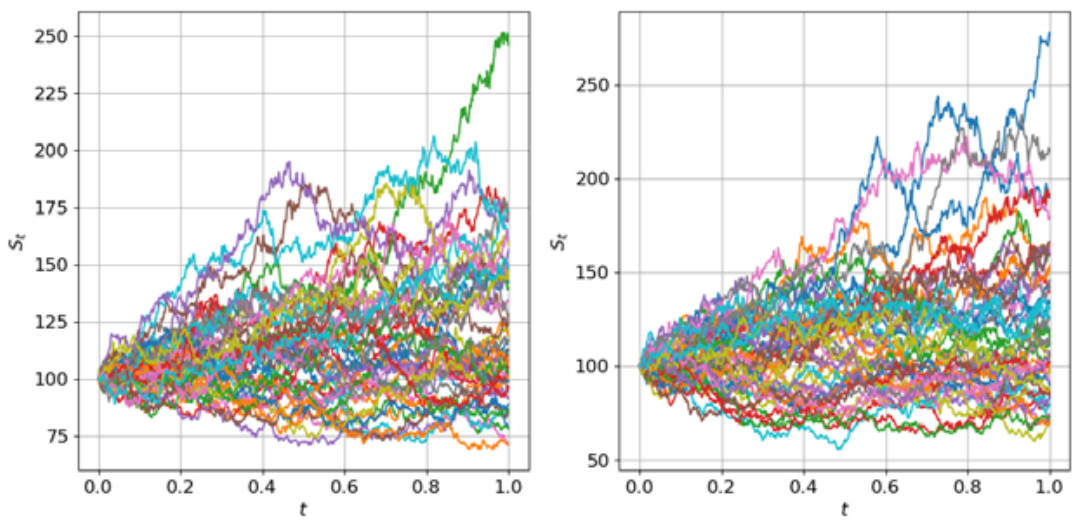

\subsection{Valoración de activos contingentes}

Consideramos ahora el problema de la valoración de un activo contingente $V \equiv V\left(t, S_{t}\right)$ con vencimiento en $T>0$ y $V\left(T, S_{T}\right)=\Phi\left(S_{T}\right)$, pactado sobre un activo riesgoso cuyo precio satisface la ecuación diferencial estocástica (18). Aplicando el lema de Itô se tiene que:

$$
d V=\left(\frac{\partial V}{\partial t}+\hat{\alpha} \frac{\partial V}{\partial S_{t}}+\frac{1}{2} \hat{\sigma}^{2} \frac{\partial^{2} V}{\partial S_{t}^{2}}\right) d t+\hat{\sigma} \frac{\partial V}{\partial S_{t}} d W_{t}
$$

Si el portafolio de cobertura autofinanciado está compuesto por $f_{t}$ unidades del activo riesgoso y $\gamma_{t}$ unidades del activo contingente, se tiene que: 


$$
d \Pi_{t}=f_{t} d S_{t}+\gamma_{t} d V
$$

De las expresiones (26) y (27) se sigue que:

$$
d \Pi_{t}=\hat{\alpha}\left(f_{t}+\gamma_{t} \frac{\partial V}{\partial S_{t}}\right) d t+\hat{\sigma}\left(f_{t}+\gamma_{t} \frac{\partial V}{\partial S_{t}}\right) d W_{t}+\gamma_{t}\left(\frac{\partial V}{\partial t}+\frac{1}{2} \hat{\sigma}^{2} \frac{\partial^{2} V}{\partial S_{t}^{2}}\right) d t
$$

Considerando nuevamente que el agente sigue una estrategia de negociación que le permita cubrir su posición en una unidad del activo contingente, tomando $\gamma_{t}=1 \mathrm{y} f_{t}=-\frac{\partial V}{\partial S_{t}}$, la ecuación (28) se transforma en:

$$
d \Pi_{t}=\left(\frac{\partial V}{\partial t}+\frac{1}{2} \hat{\sigma}^{2} \frac{\partial^{2} V}{\partial S_{t}^{2}}\right) d t
$$

expresión que por no arbitraje debe ser igual al resultado de invertir el valor inicial del portafolio a la tasa libre de riesgo, es decir:

$$
d \Pi_{t}^{(r)}=\left(-\frac{\partial V}{\partial S_{t}} S_{t}+V\right) r d t
$$

Igualando las expresiones (29) y (30) se tiene la ecuación diferencial parcial no lineal de valoración:

$$
\frac{\partial V}{\partial t}+r S_{t} \frac{\partial V}{\partial S_{t}}+\frac{1}{2} \hat{\sigma}^{2} \frac{\partial^{2} V}{\partial S_{t}^{2}}-r V=0
$$

junto con la condición de frontera $V\left(T, S_{T}\right)=\Phi\left(S_{T}\right)$. De la ecuación (17) tenemos que $\hat{\sigma}=\frac{\sigma S_{t}}{1-\lambda S_{t} \frac{\partial f}{\partial S_{t}}}$, luego:

$$
\frac{\partial V}{\partial t}+r S_{t} \frac{\partial V}{\partial S_{t}}+\frac{1}{2} \frac{\sigma^{2} S_{t}^{2}}{\left(1-\lambda S_{t} \frac{\partial f}{\partial S_{t}}\right)^{2}} \frac{\partial^{2} V}{\partial S_{t}^{2}}-r V=0
$$

y como $f \equiv f_{t}=-\frac{\partial V}{\partial S_{t}}$, entonces $-\frac{\partial f}{\partial S_{t}}=\frac{\partial^{2} V}{\partial S_{t}^{2}}$, y la EDP de valoración es:

$$
\frac{\partial V}{\partial t}+r S_{t} \frac{\partial V}{\partial S_{t}}+\frac{1}{2}\left[\frac{\sigma^{2} S_{t}^{2}}{\left(1-\lambda S_{t} \frac{\partial^{2} V}{\partial S_{t}^{2}}\right)^{2}}\right] \frac{\partial^{2} V}{\partial S_{t}^{2}}-r V=0
$$


que es una EDP completamente no lineal. Una aproximación numérica clásica como el método de diferencias finitas puede resultar poco eficiente para tratar este tipo de EDP no lineal debido a problemas con la estabilidad del método o la dimensión del problema. Recientemente, se han venido desarrollado una serie de procedimientos de aproximación a la solución basados en extensiones no lineales del teorema de representación estocástica de Feynman-Kac y redes neuronales profundas, que han mostrado ser eficientes para este tipo de problemas.

\subsection{Estrategias con componente de aleatoriedad}

Consideremos ahora el caso en el cual la estrategia de negociación del agente es descrita por un proceso de difusión de la forma (Liu \& Yong, 2005):

$$
d f\left(t, S_{t}\right)=\eta_{t} d t+\xi_{t} d W_{t} \quad ; \quad f\left(0, S_{0}\right)=f_{0}
$$

donde: $\eta_{t}$ y $\xi_{t}$ son endogenamente determinados, $W_{t}$ un movimiento Browniano estándar (el mismo que el asociado a la dinámica del precio del activo riesgoso), y $f_{0}$ es el número de unidades del activo en las que se toma posición en el instante $t=0$. En este caso se considera que el agente negocia cantidades del activo riesgoso con el ánimo de mantener la cobertura de su posición, pero su estrategia está influenciada por choques aleatorios asociados a los movimientos del precio del activo.

De acuerdo con esto, la dinámica de los precios es:

$$
\begin{aligned}
d S_{t} & =\alpha S_{t} d t+\sigma S_{t} d W_{t}+\lambda S_{t} d f\left(t, S_{t}\right) \\
& =\alpha S_{t} d t+\sigma S_{t} d W_{t}+\lambda S_{t}\left(\eta_{t} d t+\xi_{t} d W_{t}\right) \\
& =\underbrace{\left(\alpha+\lambda \eta_{t}\right)}_{\tilde{\alpha}} S_{t} d t+\underbrace{\left(\sigma+\lambda \xi_{t}\right)}_{\tilde{\sigma}} S_{t} d W_{t}
\end{aligned}
$$

es decir: 


$$
d S_{t}=\tilde{\alpha} S_{t} d t+\tilde{\sigma} S_{t} d W_{t}
$$

La figura 3 muestra trayectorias simuladas del precio del activo riesgoso bajo el esquema descrito por la expresión (35), asumiendo que los coeficientes del proceso de negociación $\eta_{t}$ y $\xi_{t}$ son constantes con $\xi>0$. Las figuras corroboran el efecto de la estrategia de negociación del agente sobre los precios. Un valor de $\eta$ positivo empuja los precios al alza, mientras que un valor negativo los empuja a la baja. De otro lado, un valor de $\xi>0$ genera una mayor volatilidad, lo que es de esperarse dado que el coeficiente de difusión del proceso aparece incrementado.

Figura 3: Trayectorias simuladas del proceso de precio para diferentes valores de los coeficientes $\eta$ y $\xi$
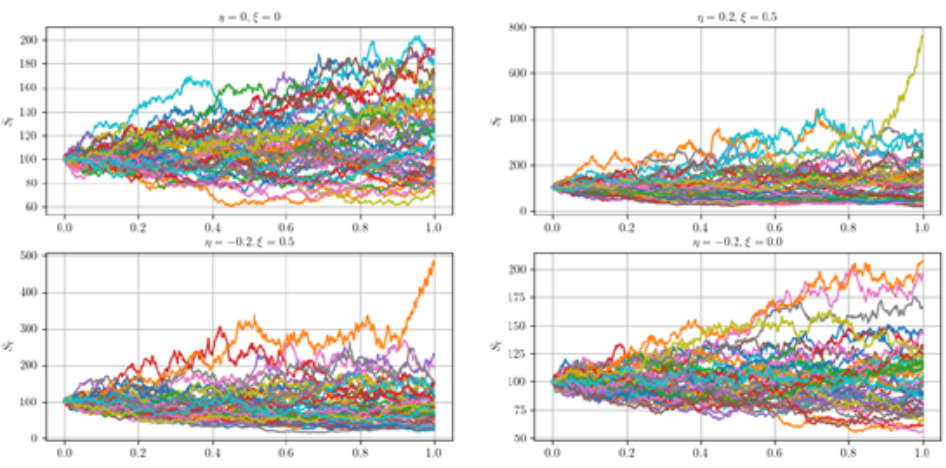

Considerando de nuevo el problema de la valoración de un activo contingente $V \equiv$ $V\left(t, S_{t}\right)$ con vencimiento en $T>0$ y $V\left(t, S_{T}\right)=\Phi\left(S_{T}\right)$, que satisface:

$$
d V=\left(\frac{\partial V}{\partial t}+\tilde{\alpha} S_{t} \frac{\partial V}{\partial S_{t}}+\frac{1}{2} \tilde{\sigma}^{2} S_{t}^{2} \frac{\partial^{2} V}{\partial S_{t}^{2}}\right) d t+\tilde{\sigma} S_{t} \frac{\partial V}{\partial S_{t}} d W_{t}
$$


se conforma un portafolio de cobertura autofinanciado $\Pi_{t}$, compuesto por $f_{t}$ unidades del activo riesgoso y $\gamma_{t}$ unidades del activo contingente; así, se sigue de las expresiones (35) y (36), junto con las expresiones para el diferencial estocástico del portafolio (27), que:

$$
d \Pi_{t}=\tilde{\alpha} S\left(f_{t}+\gamma_{t} \frac{\partial V}{\partial S_{t}}\right) d t+\tilde{\sigma} S_{t}\left(f_{t}+\gamma_{t} \frac{\partial V}{\partial S_{t}}\right) d W_{t}+\gamma_{t}\left(\frac{\partial V}{\partial t}+\frac{1}{2} \tilde{\sigma}^{2} S_{t}^{2} \frac{\partial^{2} V}{\partial S_{t}^{2}}\right) d t
$$

Si el agente sigue una estrategia de negociación que le permita cubrir su posición en una unidad del activo contingente tomando $\gamma_{t}=1$ y $f_{t}=-\frac{\partial V}{\partial S_{t}}$, la ecuación (37) se transforma en:

$$
d \Pi_{t}=\left(\frac{\partial V}{\partial t}+\frac{1}{2} \tilde{\sigma}^{2} S_{t}^{2} \frac{\partial^{2} V}{\partial S_{t}^{2}}\right) d t
$$

expresión que por no arbitraje debe ser igual al resultado de invertir el valor inicial del portafolio a la tasa libre de riesgo, es decir:

$$
d \Pi_{t}^{(r)}=\left(-\frac{\partial V}{\partial S_{t}} S_{t}+V\right) r d t
$$

Igualando las expresiones (38) y (39) se tiene la ecuación diferencial parcial no lineal de valoración:

$$
\frac{\partial V}{\partial t}+r S_{t} \frac{\partial V}{\partial S_{t}}+\frac{1}{2} \tilde{\sigma}^{2} S_{t}^{2} \frac{\partial^{2} V}{\partial S_{t}^{2}}-r V=0
$$

junto con la condición de frontera $V\left(T, S_{T}\right)=\Phi\left(S_{T}\right)$. Como $\tilde{\sigma}=\sigma+\lambda \xi_{t}$ se sigue que la EDP de valoración es:

$$
\frac{\partial V}{\partial t}+r S_{t} \frac{\partial V}{\partial S_{t}}+\frac{1}{2}\left(\sigma+\lambda \xi_{t}\right)^{2} S_{t}^{2} \frac{\partial^{2} V}{\partial S_{t}^{2}}-r V=0
$$




\section{Modelo de mercado con liquidez estocástica}

Consideramos ahora un mercado en el cual el factor de liquidez del mercado, $\lambda\left(t, S_{t}\right)$, es estocástico. Este modelo no solo permite considerar el efecto de las estrategias de negociación de los agentes en los precios y viceversa, también captura situaciones en las cuales la liquidez del mercado se puede ver afectada por choques aleatorios como cambios inesperados de regulación, en las tendencias e indicadores macroeconómicos, en la disposición de recursos, cierres forzados de la actividad económica (como en el caso de las medidas de cuarentena tomadas para controlar enfermedades), entre otros.

En este caso:

$$
d S_{t}=\alpha S_{t} d t+\sigma S_{t} d W_{t}^{(S)}+\lambda_{t} S_{t} d f_{t}
$$

donde: $\lambda_{t} \equiv \lambda\left(t, S_{t}\right), f_{t}=f\left(t, S_{t}\right)$. Se asume, además, que el factor de liquidez es descrito por un proceso CIR de la forma que:

$$
d \lambda_{t}=\kappa\left(\theta-\lambda_{t}\right) d t+\nu \sqrt{\lambda_{t}} d W_{t}^{(\lambda)}
$$

con $d W_{t}^{(S)} d W_{t}^{(\lambda)}=\rho d t$, donde $\rho$ es una constante que mide la correlación entre los movimientos Brownianos $W_{t}^{(S)}$ y $W_{t}^{(\lambda)}$. En este caso: $\theta$ es un nivel de liquidez constante de largo plazo, $\kappa$ es la velocidad de reversión al nivel $\theta$, y $\nu>0$ es la volatilidad de la liquidez. Este tipo de proceso implica que $\lambda_{t}>0$ para todos los valores positivos de $\kappa$ y $\theta$, y se garantiza que $\lambda_{t}$ no será igual a cero si se tiene que $2 \kappa \theta \geq \nu^{2}$.

De acuerdo con la expresión (42) para garantizar precios positivos se debe acotar $\lambda_{t}$ superiormente, es decir, $\lambda_{t} \leq \hat{\lambda}$ para todo $t$, de forma que $\hat{\lambda} \Delta f>-1$.

Sean $W_{t}^{1}$ y $W_{t}^{2}$ dos movimientos Brownianos estándar independientes. Tomando

$$
W_{t}^{(S)}=W_{t}^{1} \quad ; \quad W_{t}^{(\lambda)}=\rho W_{t}^{1}+\sqrt{1-\rho^{2}} W_{t}^{2}
$$

se tiene que: 


$$
\begin{gathered}
d S_{t}=\alpha S_{t} d t+\sigma S_{t} d W_{t}^{1}+\lambda_{t} S_{t} d f_{t} \\
d \lambda_{t}=\kappa\left(\theta-\lambda_{t}\right) d t+\nu \sqrt{\lambda_{t}} \rho d W_{t}^{1}+\nu \sqrt{\lambda_{t}} \sqrt{1-\rho^{2}} d W_{t}^{2}
\end{gathered}
$$

Asumiendo que $f \equiv f\left(t, S_{t}, \lambda_{t}\right) \in C^{1,2,2}$, entonces, aplicando el lema de Itô:

$$
\begin{aligned}
d f & =\frac{\partial f}{\partial t} d t+\frac{\partial f}{\partial S_{t}} d S_{t}+\frac{\partial f}{\partial \lambda_{t}} d \lambda_{t}+\frac{1}{2}\left[\frac{\partial^{2} f}{\partial S_{t}^{2}}\left(d S_{t}\right)^{2}+\frac{\partial^{2} f}{\partial \lambda_{t}^{2}}\left(d \lambda_{t}\right)^{2}+2 \frac{\partial^{2} f}{\partial S_{t} \partial \lambda_{t}}\left(d S_{t}\right)\left(d \lambda_{t}\right)\right] \\
& =\frac{\partial f}{\partial t} d t+\frac{\partial f}{\partial S_{t}} d S_{t}+\frac{\partial f}{\partial \lambda_{t}} d \lambda_{t}+\frac{\partial^{2} f}{\partial S_{t} \partial \lambda_{t}}\left(d S_{t}\right)\left(d \lambda_{t}\right)+\frac{1}{2}\left[\frac{\partial^{2} f}{\partial S_{t}^{2}}\left(d S_{t}\right)^{2}+\frac{\partial^{2} f}{\partial \lambda_{t}^{2}}\left(d \lambda_{t}\right)^{2}\right]
\end{aligned}
$$

y de la expresión (46) se sigue que:

$$
\left(d \lambda_{t}\right)^{2}=\nu^{2} \lambda_{t} d t
$$

entonces,

$$
\begin{gathered}
d f=\frac{\partial f}{\partial t} d t+\frac{\partial f}{\partial S_{t}} d S_{t}+\frac{\partial f}{\partial \lambda_{t}}\left[\kappa\left(\theta-\lambda_{t}\right) d t+\nu \sqrt{\lambda_{t}} \rho d W_{t}^{1}+\nu \sqrt{\lambda_{t}} \sqrt{1-\rho^{2}} d W_{t}^{2}\right] \\
+\frac{\partial^{2} f}{\partial S_{t} \partial \lambda_{t}}\left(d S_{t}\right)\left(d \lambda_{t}\right)+\frac{1}{2}\left[\frac{\partial^{2} f}{\partial S_{t}^{2}}\left(d S_{t}\right)^{2}+\frac{\partial^{2} f}{\partial \lambda_{t}^{2}} \nu^{2} \lambda_{t} d t\right]
\end{gathered}
$$

Como:

$$
d S_{t}=\alpha S_{t} d t+\sigma S_{t} d W_{t}^{1}+\lambda_{t} S_{t} d f_{t}
$$

reemplazando la expresión (49) y haciendo algunas simplificaciones algebraicas, se tiene que:

$$
\begin{aligned}
d S_{t} & =\left(\sigma+\lambda_{t} \nu \sqrt{\lambda_{t}} \rho \frac{\partial f}{\partial \lambda_{t}}\right) S_{t} d W_{t}^{1}+\lambda_{t} S_{t} \nu \sqrt{\lambda_{t}} \sqrt{1-\rho^{2}} \frac{\partial f}{\partial \lambda_{t}} d W_{t}^{2} \\
& +\lambda_{t} S_{t}\left(\frac{\alpha}{\lambda_{t}}+\frac{\partial f}{\partial t}\right) d t+\lambda_{t} S_{t} \frac{\partial f}{\partial S_{t}} d S_{t}+\lambda_{t} S_{t} \frac{\partial f}{\partial \lambda_{t}} \kappa\left(\theta-\lambda_{t}\right) d t \\
& +\lambda_{t} S_{t} \frac{\partial^{2} f}{\partial S_{t} \partial \lambda_{t}}\left(d S_{t}\right)\left(d \lambda_{t}\right)+\lambda_{t} S_{t} \frac{1}{2} \frac{\partial^{2} f}{\partial S_{t}^{2}}\left(d S_{t}\right)^{2}+\lambda_{t} S_{t} \frac{1}{2} \frac{\partial^{2} f}{\partial \lambda_{t}^{2}} \nu^{2} \lambda_{t} d t
\end{aligned}
$$


de donde se sigue que:

$$
\begin{aligned}
d S_{t} & =\frac{1}{1-\lambda_{t} S_{t} \frac{\partial f}{\partial S_{t}}}\left\{\lambda _ { t } S _ { t } \left[\left(\frac{\alpha}{\lambda_{t}}+\frac{\partial f}{\partial t}+\kappa\left(\theta-\lambda_{t}\right) \frac{\partial f}{\partial \lambda_{t}}+\frac{1}{2} \frac{\partial^{2} f}{\partial \lambda_{t}^{2}} \nu^{2} \lambda_{t}\right) d t\right.\right. \\
& \left.\left.+\frac{\partial^{2} f}{\partial S_{t} \partial \lambda_{t}}\left(d S_{t}\right)\left(d \lambda_{t}\right)+\frac{1}{2} \frac{\partial^{2} f}{\partial S_{t}^{2}}\left(d S_{t}\right)^{2}\right]\right\}+\left(\frac{\sigma+\lambda_{t} \nu \sqrt{\lambda_{t}} \rho \frac{\partial f}{\partial \lambda_{t}}}{1-\lambda_{t} S_{t} \frac{\partial f}{\partial S_{t}}}\right) S_{t} d W_{t}^{1} \\
& +\left(\frac{\lambda_{t} \nu \sqrt{\lambda_{t}} \sqrt{1-\rho^{2}} \frac{\partial f}{\partial \lambda_{t}}}{1-\lambda_{t} S_{t} \frac{\partial f}{\partial S_{t}}}\right) S_{t} d W_{t}^{2}
\end{aligned}
$$

\section{Denotando por:}

$$
\nu_{1}\left(t, S_{t}, \lambda_{t}\right) \equiv \nu_{1}:=\left(\frac{\sigma+\lambda_{t} \nu \sqrt{\lambda_{t}} \rho \frac{\partial f}{\partial \lambda_{t}}}{1-\lambda_{t} S_{t} \frac{\partial f}{\partial S_{t}}}\right)
$$

y

$$
\nu_{2}\left(t, S_{t}, \lambda_{t}\right) \equiv \nu_{2}:=\left(\frac{\lambda_{t} \nu \sqrt{\lambda_{t}} \sqrt{1-\rho^{2}} \frac{\partial f}{\partial \lambda_{t}}}{1-\lambda_{t} S_{t} \frac{\partial f}{\partial S_{t}}}\right)
$$

se tiene que la expresión (51) se reescribe como:

$$
\begin{aligned}
d S_{t} & =\frac{1}{1-\lambda_{t} S_{t} \frac{\partial f}{\partial S_{t}}}\left\{\lambda _ { t } S _ { t } \left[\left(\frac{\alpha}{\lambda_{t}}+\frac{\partial f}{\partial t}+\kappa\left(\theta-\lambda_{t}\right) \frac{\partial f}{\partial \lambda_{t}}+\frac{1}{2} \frac{\partial^{2} f}{\partial \lambda_{t}^{2}} \nu^{2} \lambda_{t}\right) d t\right.\right. \\
& \left.\left.+\frac{\partial^{2} f}{\partial S_{t} \partial \lambda_{t}}\left(d S_{t}\right)\left(d \lambda_{t}\right)+\frac{1}{2} \frac{\partial^{2} f}{\partial S_{t}^{2}}\left(d S_{t}\right)^{2}\right]\right\}+\nu_{1} S_{t} d W_{t}^{1}+\nu_{2} S_{t} d W_{t}^{2}
\end{aligned}
$$

De (54) se sigue que:

$$
\left(d S_{t}\right)^{2}=S_{t}^{2}\left(\nu_{1}^{2}+\nu_{2}^{2}\right) d t=\frac{S_{t}^{2}}{1-\lambda_{t} S_{t} \frac{\partial f}{\partial S_{t}}}\left[\sigma^{2}+2 \sigma \lambda_{t} \rho \nu \sqrt{\lambda_{t}} \frac{\partial f}{\partial \lambda_{t}}+\lambda_{t}^{3} \nu^{2}\left(\frac{\partial f}{\partial \lambda_{t}}\right)^{2}\right]
$$

Como tenemos que:

$$
\begin{aligned}
d S_{t} & =\frac{\lambda_{t} S_{t}}{1-\lambda_{t} S_{t} \frac{\partial f}{\partial S_{t}}}\left(\frac{\alpha}{\lambda_{t}}+\frac{\partial f}{\partial t}+\kappa\left(\theta-\lambda_{t}\right) \frac{\partial f}{\partial \lambda_{t}}+\frac{1}{2} \frac{\partial^{2} f}{\partial \lambda_{t}^{2}} \nu^{2} \lambda_{t}\right) d t \\
& \frac{1}{1-\lambda_{t} S_{t} \frac{\partial f}{\partial S_{t}}} \frac{\partial^{2} f}{\partial S_{t} \partial \lambda_{t}}\left(d S_{t}\right)\left(d \lambda_{t}\right)+\frac{1}{1-\lambda_{t} S_{t} \frac{\partial f}{\partial S_{t}}} \frac{1}{2} \frac{\partial^{2} f}{\partial S_{t}^{2}}\left(d S_{t}\right)^{2}+\nu_{1} S_{t} d W_{t}^{1}+\nu_{2} S_{t} d W_{t}^{2}
\end{aligned}
$$


y

$$
d \lambda_{t}=\kappa\left(\theta-\lambda_{t}\right) d t+\nu \sqrt{\lambda_{t}} \rho d W_{t}^{1}+\nu \sqrt{\lambda_{t}} \sqrt{1-\rho^{2}} d W_{t}^{2}
$$

entonces,

$$
d S_{t} d \lambda_{t}=S_{t} \nu \sqrt{\lambda_{t}}\left(\nu_{1} \rho+\nu_{2} \sqrt{1-\rho^{2}}\right) d t=S_{t} \nu \sqrt{\lambda_{t}}\left(\frac{\sigma \rho+\lambda_{t} \nu \sqrt{\lambda_{t}} \frac{\partial f}{\partial \lambda_{t}}}{1-\lambda_{t} S_{t} \frac{\partial f}{\partial S_{t}}}\right) d t
$$

Los anteriores resultados se resumen en la siguiente proposición.

Proposición 1. En un mercado con liquidez estocástica descrita por un proceso CIR de la forma:

$$
d \lambda_{t}=\kappa\left(\theta-\lambda_{t}\right) d t+\nu \sqrt{\lambda_{t}} \rho d W_{t}^{1}+\nu \sqrt{\lambda_{t}} \sqrt{1-\rho^{2}} d W_{t}^{2}
$$

y denotando por:

$$
\begin{gathered}
\nu_{1}:=\frac{\sigma}{1-\lambda_{t} S_{t} \frac{\partial f}{\partial S_{t}}}+\rho \frac{\lambda_{t} \nu \sqrt{\lambda_{t}} \rho \frac{\partial f}{\partial \lambda_{t}}}{1-\lambda_{t} S_{t} \frac{\partial f}{\partial S_{t}}} \\
\nu_{2}:=\sqrt{1-\rho^{2}}\left(\frac{\lambda_{t} \nu \sqrt{\lambda_{t}} \frac{\partial f}{\partial \lambda_{t}}}{1-\lambda_{t} S_{t} \frac{\partial f}{\partial S_{t}}}\right) \\
b\left(t, S_{t}, \lambda_{t}\right):=\frac{\lambda_{t}}{1-\lambda_{t} S_{t} \frac{\partial f}{\partial S_{t}}}\left[\frac{\alpha}{\lambda_{t}}+\frac{\partial f}{\partial t}+\kappa\left(\theta-\lambda_{t}\right) \frac{\partial f}{\partial \lambda_{t}}+\frac{1}{2} \frac{\partial^{2} f}{\partial \lambda_{t}^{2}} \nu^{2} \lambda_{t}\right. \\
\left.+\frac{1}{2} \frac{\partial^{2} f}{\partial S_{t}^{2}} S_{t}^{2}\left(\nu_{1}^{2}+\nu_{2}^{2}\right)+\frac{\partial^{2} f}{\partial S_{t} \partial \lambda_{t}} S_{t} \nu \sqrt{\lambda_{t}}\left(\nu_{1} \rho+\nu_{2} \sqrt{1-\rho^{2}}\right)\right]
\end{gathered}
$$

la ecuación diferencial estocástica que describe la dinámica de los precios de los activos riesgosos es:

$$
d S_{t}=S_{t} b\left(t, S_{t}, \lambda_{t}\right)+S_{t} \nu_{1} d W_{t}^{1}+S_{t} \nu_{2} d W_{t}^{2}
$$

donde: $W_{t}^{1} y W_{t}^{2}$ son movimientos Brownianos estándar independientes y $f\left(t, S_{t}\right)$ es la estrategia de negociación del agente. 
Se debe resaltar que en este caso, como lo muestra la ecuación (63), la EDE para la dinámica de los precios de los activos riesgosos incorpora dos fuentes de aleatoriedad cuyos coeficientes están asociados vía el factor de correlación $\rho$.

Siguiendo la misma metodología de valoración por replicación presentada en las secciones anteriores, el problema de valorar un activo contingente $V \equiv V\left(t, S_{t}\right)$ donde el precio del activo subyacente $\left(S_{t}\right)$ satisface la EDE (63), con vencimiento en $T>0$ y $V\left(T, S_{t}\right)=\Phi\left(S_{T}\right)$, implica considerar un portafolio $\Pi_{t}$ compuesto por $f_{t}$ unidades del activo riesgoso y $\gamma_{t}$ unidades del activo contingente. Se tiene entonces que:

$$
\Pi_{t}=f_{t} S_{t}+\gamma_{t} V
$$

y por autofinanciamiento:

$$
d \Pi_{t}=f_{t} d S_{t}+\gamma_{t} d V
$$

Aplicando la fórmula de Itô a $V\left(t, S_{t}\right)$ se tiene:

$$
d V=\frac{\partial V}{\partial t} d t+\frac{\partial V}{\partial S_{t}} d S_{t}+\frac{1}{2} \frac{\partial^{2} V}{\partial S_{t}^{2}}\left(d S_{t}\right)^{2}
$$

reemplazando las expresiones (63) y (55), y al hacer $b \equiv b\left(t, S_{t}, \lambda_{t}\right)$, la expresión (66) se transforma en:

$$
d V=\left(\frac{\partial V}{\partial t}+b S_{t} \frac{\partial V}{\partial S_{t}}+\frac{1}{2} S_{t}^{2}\left(\nu_{1}^{2}+\nu_{2}^{2}\right) \frac{\partial^{2} V}{\partial S_{t}^{2}}\right) d t+S_{t} \nu_{1} \frac{\partial V}{\partial S_{t}} d W_{t}^{1}+S_{t} \nu_{2} \frac{\partial V}{\partial S_{t}} d W_{t}^{2}
$$

y la dinámica del portafolio es:

$$
\begin{aligned}
d \Pi_{t} & =b S_{t}\left(f_{t}+\gamma_{t} \frac{\partial V}{\partial S_{t}}\right) d t+S_{t} \nu_{1}\left(f_{t}+\gamma_{t} \frac{\partial V}{\partial S_{t}}\right) d W_{t}^{1} \\
& +S_{t} \nu_{2}\left(f_{t}+\gamma_{t} \frac{\partial V}{\partial S_{t}}\right) d W_{t}^{2}+\gamma_{t}\left(\frac{\partial V}{\partial t}+\frac{1}{2} S_{t}^{2}\left(\nu_{1}^{2}+\nu_{2}^{2}\right) \frac{\partial^{2} V}{\partial S_{t}^{2}}\right) d t
\end{aligned}
$$


Considerando una cobertura delta, $f_{t}=\frac{\partial V}{\partial S_{t}}$ y $\gamma_{t}=-1$, entonces:

$$
d \Pi_{t}=\left(\frac{\partial V}{\partial t}+\frac{1}{2} S_{t}^{2}\left(\nu_{1}^{2}+\nu_{2}^{2}\right) \frac{\partial^{2} V}{\partial S_{t}^{2}}\right) d t
$$

expresión que por no arbitraje debe ser igual a la dinámica de invertir el valor inicial del portafolio a la tasa libre de riesgo $r$, es decir:

$$
d \Pi_{t}^{(r)}=\left(-\frac{\partial V}{\partial S_{t}} S_{t}+V\right) r d t
$$

Igualando las expresiones (69) y (70) se tiene la EDP de valoración:

$$
\frac{\partial V}{\partial t}+r S_{t} \frac{\partial V}{\partial S_{t}}+\frac{1}{2} S_{t}^{2}\left(\nu_{1}^{2}+\nu_{2}^{2}\right) \frac{\partial^{2} V}{\partial S_{t}^{2}}-r V=0
$$

con $V\left(T, S_{T}\right)=\Phi\left(S_{T}\right)$. Dado que:

$$
\nu_{1}^{2}+\nu_{2}^{2}=\frac{\sigma^{2}+2 \sigma \rho \lambda_{t} \nu \sqrt{\lambda_{t}}\left(\frac{\partial f}{\partial \lambda_{t}}\right)+\lambda_{t}^{3} \nu^{2}\left(\frac{\partial f}{\partial \lambda_{t}}\right)^{2}}{\left(1-\lambda_{t} S_{t} \frac{\partial f}{\partial S_{t}}\right)^{2}}
$$

la EDP de valoración se reescribe como:

$$
\frac{\partial V}{\partial t}+r S_{t} \frac{\partial V}{\partial S_{t}}+\frac{1}{2} S_{t}^{2}\left(\frac{\sigma^{2}+2 \sigma \rho \lambda_{t} \nu \sqrt{\lambda_{t}}\left(\frac{\partial f}{\partial \lambda_{t}}\right)+\lambda_{t}^{3} \nu^{2}\left(\frac{\partial f}{\partial \lambda_{t}}\right)^{2}}{\left(1-\lambda_{t} S_{t} \frac{\partial f}{\partial S_{t}}\right)^{2}}\right) \frac{\partial^{2} V}{\partial S_{t}^{2}}-r V=0
$$

Como la estrategia considerada es $f_{t}=\frac{\partial V}{\partial S_{t}}$, entonces $\frac{\partial f_{t}}{\partial S_{t}}=\frac{\partial^{2} V}{\partial S_{t}^{2}}$ y $\frac{\partial f_{t}}{\partial \lambda_{t}}=\frac{\partial^{2} V}{\partial \lambda_{t} \partial S_{t}}$, y la EDP de valoración es, finalmente:

$$
\frac{\partial V}{\partial t}+r S_{t} \frac{\partial V}{\partial S_{t}}+\frac{1}{2} S_{t}^{2}\left(\frac{\sigma^{2}+2 \sigma \rho \lambda_{t} \nu \sqrt{\lambda_{t}}\left(\frac{\partial^{2} V}{\partial \lambda_{t} \partial S_{t}}\right)+\lambda_{t}^{3} \nu^{2}\left(\frac{\partial^{2} V}{\partial \lambda_{t} \partial S_{t}}\right)^{2}}{\left(1-\lambda_{t} S_{t} \frac{\partial^{2} V}{\partial S_{t}^{2}}\right)^{2}}\right) \frac{\partial^{2} V}{\partial S_{t}^{2}}-r V=0
$$

que es una EDP de segundo orden completamente no lineal. 


\section{Conclusiones y extensiones}

La dinámica de los mercados financieros globales incorpora de forma natural un riesgo por liquidez, esto debido a la disponibilidad limitada en tiempo y volumen de los activos necesarios para el desarrollo de estrategias de negociación. En este documento se considera este riesgo al incorporar un factor de liquidez del mercado, modelado a partir de la relación que se puede establecer entre la dinámica de los precios de los activos riesgosos y las estrategias de negociación de los agentes.

En los casos considerados en este trabajo se modela de forma explícita el efecto de la estrategia de negociación del agente sobre los componentes del proceso de precios. En particular, en el caso de un mercado con liquidez estocástica, la ecuación diferencial estocástica para la dinámica de los precios incorpora dos fuentes de aleatoriedad cuyos coeficientes dependen del nivel de correlación entre las fuentes de incertidumbre del modelo de precios y del factor de liquidez, así como de la estrategia de negociación misma.

Para los diferentes escenarios considerados se establecen las ecuaciones diferenciales parciales de valoración correspondientes; en los casos en donde la liquidez no es constante se encuentra que la ecuación de valoración es una EDP de segundo orden no lineal.

Como extensiones a este trabajo se pueden plantear por lo menos dos líneas de desarrollo. Por un lado está el problema de la calibración de los parámetros de los modelos propuestos a partir de un conjunto discreto de observaciones del mercado. De otra parte está la resolución de las EDP de valoración, problema que no resulta fácil de tratar dado su carácter no lineal. 


\section{Referencias}

Acharya, V. V. \& Pedersen, L. H. (2005). Asset pricing with liquidity risk. Journal of Financial Economics, 77(2), 375-410.

Amihud, Y. (2002). Illiquidity and stock returns: Cross-section and time-series effects. Journal of Financial Markets, 5(1), 31-56.

Amihud, Y. \& Mendelson, H. (1986). Asset pricing and the bid-ask spread. Journal of Financial Economics, 17(2), 223-249.

Black, F. \& Scholes, M. (1973). The pricing of options and corporate liabilities. Journal of Political Economy, 81(3), 637-654.

Cetin, U., Jarrow, R. A., \& Protter, P. (2010). Liquidity risk and arbitrage pricing theory. In Handbook of Quantitative Finance and Risk Management (pp. 1007-1024). Springer.

Chordia, T., Roll, R., \& Subrahmanyam, A. (2000). Commonality in liquidity. Journal of Financial Economics, 56(1), 3-28.

Chordia, T., Roll, R., \& Subrahmanyam, A. (2001). Market liquidity and trading activity. The Journal of Finance, 56(2), 501-530.

Liu, H. \& Yong, J. (2005). Option pricing with an illiquid underlying asset market. Journal of Economic Dynamics and Control, 29(12), 2125-2156.

Lo, A. W., Mamaysky, H., \& Wang, J. (2004). Asset prices and trading volume under fixed transactions costs. Journal of Political Economy, 112(5), 1054-1090.

Merton, R. C. \& Samuelson, P. A. (1990). Continuous-time finance.

Pástor, L. \& Stambaugh, R. F. (2003). Liquidity risk and expected stock returns. Journal of Political Economy, 111(3), 642-685. 
Ronnie Sircar, K. \& Papanicolaou, G. (1998). General black-scholes models accounting for increased market volatility from hedging strategies. Applied Mathematical Finance, 5(1), 45-82.

Tripathi, A., Dixit, A., et al. (2019). Liquidity of financial markets: a review. Studies in Economics and Finance.

Vayanos, D. (2004). Flight to quality, flight to liquidity, and the pricing of risk. Technical report, National bureau of economic research.

Wilmott, P. \& Schönbucher, P. J. (2000). The feedback effect of hedging in illiquid markets. SIAM Journal on Applied Mathematics, 61(1), 232-272. 